\title{
Analytic description of the radio emission of air showers based on its emission mechanisms
}

\author{
Christian Glaser ${ }^{*, a}$, Sijbrand de Jong ${ }^{b, c}$, Martin Erdmann ${ }^{a}$, Jörg R. Hörandel ${ }^{b, c}$ and \\ Erik Willems ${ }^{b}$ \\ ${ }^{a}$ RWTH Aachen University, III. Physikalisches Institut A, Aachen, Germany \\ ${ }^{b}$ IMAPP, Radboud University Nijmegen, Nijmegen, Netherlands \\ ${ }^{c}$ Nikhef, Science Park, Amsterdam, Netherlands \\ E-mail: glaseraphysik.rwth-aachen.de
}

\begin{abstract}
The spatial signal distribution of the radio frequency radiation from extensive air showers on the ground contains information on crucial cosmic-ray properties, such as energy and mass. A long standing challenge to access this information experimentally with a sparse grid of antennas is an analytic modeling of the radio signal distribution, which will be addressed in this contribution. We present an analytic model based on the two physical processes generating radio emission in air showers: the geomagnetic and the charge-excess emission. Our study is based on full Monte Carlo simulations with the CoREAS code. Besides an improved theoretical understanding of radio emission, our model describes the radio signal distribution with unprecedented precision. Our model explicitly includes polarization information, which basically doubles the information that is used from a single radio station. Hence, more precise information can be gained with an even smaller antenna multiplicity. Furthermore, exploiting our model will increase the accuracy of an independent determination of the cosmic-ray energy scale with the radio technique. We will present our model and discuss the dependence on the air-shower parameters.
\end{abstract}

35th International Cosmic Ray Conference - ICRC2017

10-20 July, 2017

Bexco, Busan, Korea

${ }^{*}$ Speaker. 


\section{Introduction}

Ultra-high energy cosmic rays can be detected by broadband radio emission from air showers. Two main emission mechanisms have been identified: The geomagnetic emission, where charged particles are deflected in the geomagnetic field, and the charge-excess emission, which is due to a time-varying negative charge-excess in the shower front.

The spatial distribution of the energy fluence, i.e. the energy per unit area of the radio electricfield pulse, holds information on relevant air shower parameters like the energy and the position of the shower maximum $X_{\max }$. The amount of energy emitted in the form of radio emission by the air shower - referred to as the radiation energy - is given by the spatial integral over the energyfluence. The radiation energy is directly related to the electromagnetic shower energy $E_{\mathrm{em}}$ and allows for a precise measurement of it with a theoretical energy resolution of only $3 \%$ [1]. Thus, the radiation energy serves as a universal estimator of the cosmic-ray energy and is exploited by the Pierre Auger Collaboration to measure cosmic-ray energies [2, 3]. In particular, this method potentially reduces the systematic uncertainties of the energy measurement as absorption of radio waves in the atmosphere is negligible and the radio emission can be calculated from first principles.

The shape of the spatial signal distribution is primarily determined by the distance $D_{\mathrm{X}_{\max }}$ from the observer to the emission region. The emission region can be approximated by the position of the shower maximum $X_{\max }$ [1] which is an estimator of the cosmic-ray mass. The usage of $D_{\mathrm{X}_{\max }}$ has the advantage that a universal description of the radio signal distribution can be given that does not depend on the specific altitude of the experiment.

A long standing challenge to access this information experimentally with a sparse grid of antennas is an analytic modeling of the radio signal distribution and will be addressed in this contribution. In [4], an empirical parametrization for the spatial radio signal distribution is introduced based on morphological arguments, which gives an adequate description of the data measured by LOFAR and AERA and was already successfully exploited to measure cosmic-ray energies [2, 3]. However, explaining the behavior and value of the parameters of this parametrization is not straightforward, as most parameters depend on various shower features. With the knowledge gain of the past years (e.g. $[1,4]$ ), we formulate an analytic description of the spatial signal distribution directly based on its physical emission processes whose parameters depend on the air-shower parameters energy and $X_{\max }$. In addition, we explicitly take the polarization of the radio signal into account which effectively doubles the available information of each antenna station.

\section{Decomposition of radio signal into geomagnetic and charge-excess contributions}

We use the CoREAS code for the simulation of the radio emission from air showers [5]. As CoREAS is a microscopic Monte Carlo code, no emission mechanism is explicitly modeled. Instead, each shower particle is tracked and the radiation resulting from its movement is calculated from first principles using classical electrodynamics. Therefore, the contribution of the geomagnetic and charge-excess emission processes to the simulated electric field can not be differentiated. However, we can exploit the different polarization signatures of the two emission mechanisms to decompose the signal into its geomagnetic and charge-excess contribution [1]. The geomagnetic emission is polarized in the direction of the Lorentz force acting on the shower particles $\vec{v} \times \vec{B}$ 


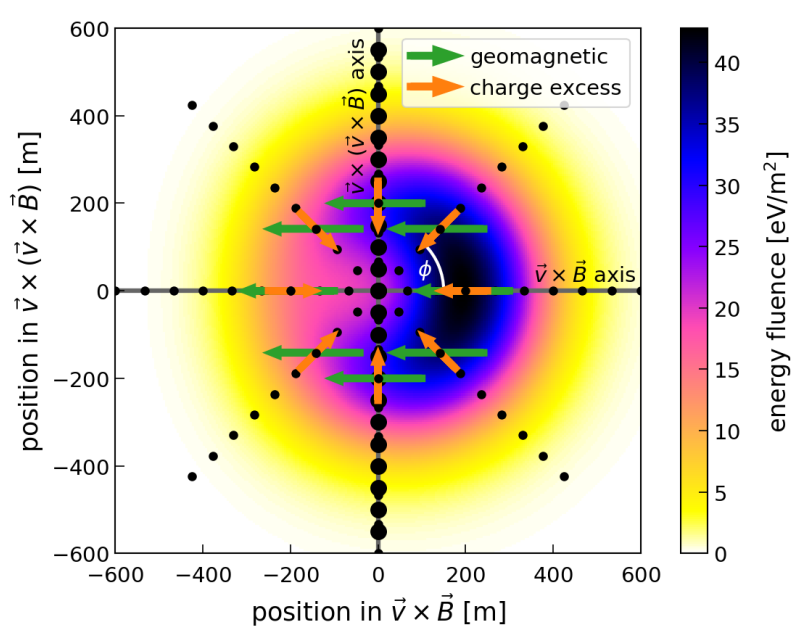

Figure 1: Distribution of the energy fluence (in the $30-80 \mathrm{MHz}$ band) of an air shower with $60^{\circ}$ zenith angle at an observation altitude of $1564 \mathrm{~m}$ asl which corresponds to the height of the Engineering Radio Array of the Pierre Auger Observatory. Superimposed is the polarization direction of the geomagnetic and charge-excess emission processes at different positions in form of arrows.

where $\vec{v}$ is the shower axis and $\vec{B}$ is the geomagnetic field. The charge-excess emission, in contrast, is polarized radially towards the shower axis. Hence, a suitable coordinate system is in the shower plane (the electric field is always polarized perpendicular to its direction of propagation $\vec{v}$ ) where one axis is aligned to the $\vec{v} \times \vec{B}$ direction and the other axis to the $\vec{v} \times(\vec{v} \times \vec{B})$ direction.

In Fig. 1, the distribution of the energy fluence is shown in the $\vec{v} \times \vec{B}-\vec{v} \times(\vec{v} \times \vec{B})$ coordinate system for a typical air shower. At observer positions on the $\vec{v} \times(\vec{v} \times \vec{B})$ axis, the polarizations of the signals from the geomagnetic and charge-excess processes are orthogonal. The $\vec{v} \times \vec{B}$ component of the electric field $E_{\vec{v} \times \vec{B}}$ originates only from geomagnetic emission, whereas the $\vec{v} \times(\vec{v} \times \vec{B})$ component of the electric field $E_{\vec{v} \times(\vec{v} \times \vec{B})}$ originates only from charge-excess emission. We calculate the energy fluence $f_{\vec{v} \times \vec{B}}$ from $E_{\vec{v} \times \vec{B}}$ and $f_{\vec{v} \times(\vec{v} \times \vec{B})}$ from $E_{\vec{v} \times(\vec{v} \times \vec{B})}$ which corresponds to $f_{\text {geo }}$ and $f_{\text {ce }}$ for observers on the $\vec{v} \times(\vec{v} \times \vec{B})$ axis.

In Fig. 2 and 3, the two components of the energy fluence $\left(f_{\vec{v} \times \vec{B}}=f_{\text {geo }}, f_{\vec{v} \times(\vec{v} \times \vec{B})}=f_{\text {ce }}\right)$ are presented as a function of the position along the $\vec{v} \times(\vec{v} \times \vec{B})$ axis of two simulated air showers with different distances $D_{\mathrm{X}_{\max }}$ from the observer to the shower maximum. The first example is at a distance to $X_{\max }$ of $361 \mathrm{~g} / \mathrm{cm}^{2}$. At this distance, the radio emission of the air shower is almost completed (98\% of the radiation energy has already been emitted) [1]. For smaller values of $D_{\mathrm{X}_{\max }}$, the distribution of the energy fluence becomes even stronger peaked around the shower axis.

The second example is for a distance $D_{\mathrm{X}_{\max }}=1024 \mathrm{~g} / \mathrm{cm}^{2}$, far away from the shower development. In particular, the change of the shape of the signal distribution between the two examples is due to free propagation of the electromagnetic waves and not because additional radio emission is created by the air shower. For large distances to $X_{\max }$, the emission is peaked in a Cherenkov cone, due to the non-unity of the refractive index of air. The opening angle of the cone depends on the air pressure at the point of emission, i.e. on the height of the emission. The peaking structure of the Cherenkov cone is smeared by the fact that the emission is in an extended longitudinal region along the shower axis. 

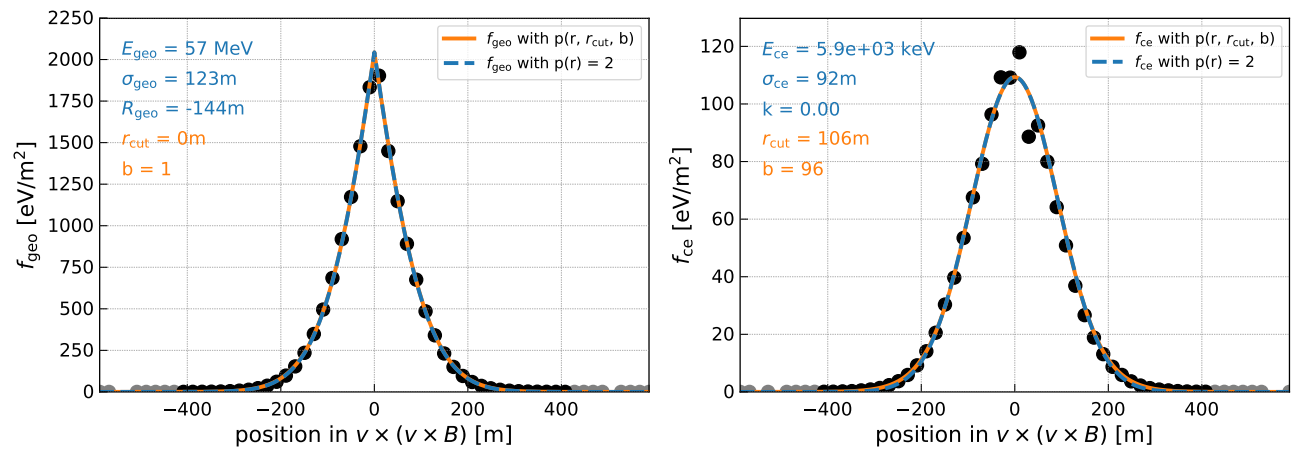

Figure 2: Energy fluence (in the $30-80 \mathrm{MHz}$ band) from geomagnetic (left) and charge-excess emission (right) along the $\vec{v} \times(\vec{v} \times \vec{B})$ axis of a $3 \mathrm{EeV}$ iron induced air shower with a zenith angle of $32^{\circ}$ observed at an altitude of $1564 \mathrm{~m}$ asl. The corresponding distance to $X_{\max }$ is $361 \mathrm{~g} / \mathrm{cm}^{2}$. Gray circles denote data points with a signal less than $10^{-4}$ of the maximum signal which are not used in the fit. The dashed line shows the best fit with $p(r)=2$ (see below Eq. (4.1) and Eq. (5.1)). The solid line denotes the best fit if the parameters $r_{\text {cut }}$ and $b$ of $p(r)$ are both varied.
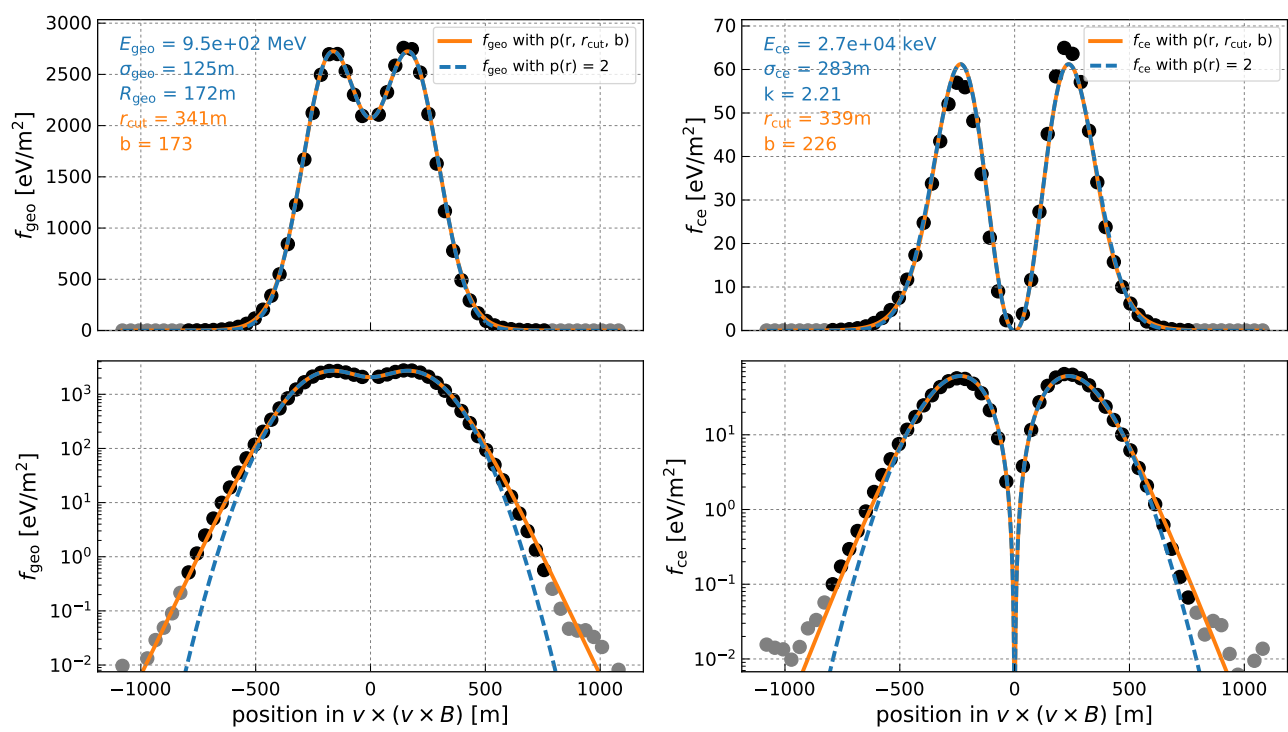

Figure 3: Same information as in Fig. 2 but for a $10 \mathrm{EeV}$ iron induced air shower with a zenith angle of $60^{\circ}$ observed at an altitude of $1564 \mathrm{~m}$ asl. The corresponding distance to $X_{\max }$ is $1024 \mathrm{~g} / \mathrm{cm}^{2}$. The upper panels are on a linear scale and the lower panels are on a logarithmic scale. The slight asymmetry can be attributed to the projection of the signal at ground onto the shower plane.

\section{Monte-Carlo data set}

For this analysis we use a set of 300 air showers simulated with CoREAS [5]. 50\% of the air showers have an iron primary and 50\% have a proton primary. The cosmic-ray energy is distributed between $10^{17} \mathrm{eV}$ to $10^{19} \mathrm{eV}$ uniformly in the logarithm of the energy. The zenith angle $\theta$ is distributed uniformly in $\cos \theta$ from $0^{\circ}$ to $60^{\circ}$ and the azimuth angle is chosen randomly. For each air shower, we calculate the radio emission for two observations planes, one at $1564 \mathrm{~m}$ as corresponding to the altitude of the Radio Array of the Pierre Auger Observatory - and another 
one at sea level - corresponding to the altitude of the LOFAR cosmic-ray radio detector. In each observation plane, the observer positions are positioned in a star pattern in the $\vec{v} \times \vec{B}$ coordinate system projected on the ground plane. This choice of antenna positions allows for a effective sampling of the $2 \mathrm{D}$ radio signal distribution and decomposition of the emission into its geomagnetic and charge-excess contributions (cf. Fig. 1 and [1,4] for more information about the choice of observer positions). The radio pulses are filtered in the $30-80 \mathrm{MHz}$ band which corresponds to the bandwidth of most cosmic-ray radio detectors [3].

\section{Signal distribution of the geomagnetic emission}

The strength of the geomagnetic emission is circularly symmetric around the shower axis and, thus, only a function of the perpendicular distance to the shower axis $r$. In the $\vec{v} \times \vec{B}$ coordinate system, $r$ is given by $r=\sqrt{x^{2}+y^{2}}$ where $x$ and $y$ denote the position in the $\vec{v} \times \vec{B}-\vec{v} \times(\vec{v} \times \vec{B})$ plane. The energy fluence of the geomagnetic emission can be parametrized as

$$
f_{\mathrm{geo}}=\left\{\begin{array}{ll}
\frac{1}{N_{R_{-}}} E_{\mathrm{geo}}^{\prime} \exp \left(-\left(\frac{r-R_{\mathrm{geo}}}{\sqrt{2} \sigma_{\mathrm{geo}}}\right)^{p(r)}\right) & \text { if } R_{\mathrm{geo}}<0 \\
\frac{1}{N_{R_{+}}} E_{\mathrm{geo}}^{\prime}\left[\exp \left(-\left(\frac{r-R_{\mathrm{geo}}}{\sqrt{2} \sigma_{\mathrm{geo}}}\right)^{p(r)}\right)+\exp \left(-\left(\frac{r+R_{\mathrm{geo}}}{\sqrt{2} \sigma_{\mathrm{geo}}}\right)^{p(r)}\right)\right] & \text { if } R_{\mathrm{geo}} \geq 0
\end{array} .\right.
$$

The parameter $R_{\text {geo }}$ can be interpreted as the radius of the Cherenkov ring, and the parameter $\sigma_{\text {geo }}$ describes the width of the function. The function $p(r)$ is a small correction to an exponent of 2 and will be discussed below. For $p(r)=2$, the two-dimensional integral over the function (which gives the radiation energy) can be calculated analytically. The constants $N_{R_{-}}$and $N_{R_{+}}$are chosen such that the parameter $E_{\mathrm{geo}}^{\prime}$ is the geomagnetic radiation energy for $p(r)=2$.

A visualization of the function is shown in Fig. 4a. Negative values of $R_{\text {geo }}$ describe the situation when the air shower has not yet emitted all radiation energy when hitting the observer. Then, the signal distribution is strongly peaked around the shower axis which is described by the falling flanks of a Gaussian function (cf. Fig. 2). Positive values of $R_{\text {geo }}$ describe the distribution of the energy fluence after the shower has emitted all its radiation energy (which is roughly at $D_{\mathrm{X}_{\max }} \approx 450 \mathrm{~g} / \mathrm{cm}^{2}$ [1]). Then, the function is the sum of two Gaussian functions centered at $R_{\text {geo }}$ and $-R_{\text {geo }}$. If the radius $R_{\text {geo }}$ becomes larger then the width $\sigma_{\text {geo }}$, the function becomes peaked at the Cherenkov ring.

As the radiation energy $E_{\text {geo }}$ can also be calculated numerically from the air-shower simulation, we first fix $E_{\text {geo }}^{\prime}$ in the fit and determine the optimal parameters $\sigma$ and $R_{\text {geo }}$ in a $\chi^{2}$ minimization. In the fit, the data points with large energy fluence are given a larger weight than those with small energy fluence to have the central part of the function described well. This is done by assigning the same absolute uncertainty to all data points. For small distances to $X_{\max }$, where the observer is still within the shower development and the parameter $R_{\text {geo }}$ is negative, the simulated signal distribution is well described by our function (cf. Fig. 2).

For larger distances of the observer to $X_{\max }$, we observe that the central part of the signal distribution is described well but the signal falloff at large distances is slightly overestimated (cf. left panels of Fig. 3). This can be modeled by a modification of the exponent of the exponential 

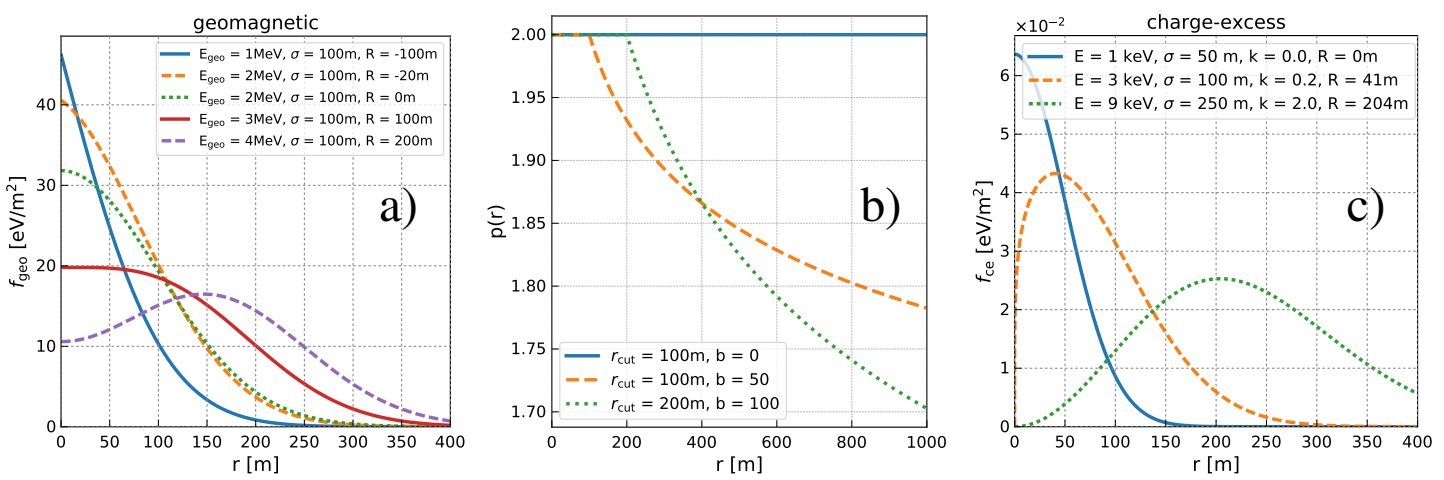

Figure 4: (left) Geomagnetic signal distribution for different parameters of our parametrization. (center) Functional form of the variation of the exponent $p(r)$ for different values of $r_{\text {cut }}$ and $b$. (right) Charge-excess signal distribution for different parameters of our parametrization.

function of Eq. (4.1) of the following form

$$
p(r)=\left\{\begin{array}{ll}
2 & \text { if } r \leq r_{\text {cut }} \\
2\left(\frac{r}{r_{\text {cut }}}\right)^{-b / 1000} & \text { if } r>r_{\text {cut }}
\end{array} \quad \text { with } r_{\text {cut }} \geq 1 \mathrm{~m}\right.
$$

The functional form of $p(r)$ is visualized for typical values of $r_{\text {cut }}$ and $b$ in Fig. 4b. For positive values of $b, p(r)$ becomes smaller than 2 for distances larger than $r_{\text {cut }}$. Hence, the signal falloff at large distances weakens. We determine the optimal parameters of $r_{\text {cut }}$ and $b$ again in a $\chi^{2}$ minimization, where we fix all other parameters $\left(E_{\mathrm{geo}}^{\prime}, \sigma_{\mathrm{geo}}\right.$ and $\left.R_{\mathrm{geo}}\right)$ and where we give all data points the same relative uncertainty. With this modification the simulated signal distribution can be described well at all distances $r$ (cf. Fig. 3).

\section{Signal distribution of the charge-excess emission}

The strength of the charge-excess emission is circular symmetric around the shower axis and can be described with a modification of the Gamma distribution

$$
f_{\mathrm{ce}}(r)=\frac{1}{N_{\mathrm{ce}}} E_{\mathrm{ce}}^{\prime} r^{k} \exp \left(\frac{-r^{p(r)}(k+1)}{p(r) \sigma_{\mathrm{ce}}^{p(r)}}\right),
$$

with $k \geq 0$. The variation of the exponent $p(r)$ has the same functional form as in the geomagnetic case (cf. Eq. (4.2)). For $k=0$ (and $p(r)=2$ ), the function is a Gaussian function with mean zero. For $k>0$ the function has the property to be zero for $r=0$. The interplay between the rising part from $r^{k}$ and the falling part from the exponential function, also models the Cherenkov ring. The distance where the function becomes maximal is given by $R_{\text {ce }}=\sigma_{c e} \sqrt{k} / \sqrt{k+1}$. The constant $N$ is chosen such that the two-dimensional integral over $f_{\mathrm{ce}}$ is $E_{\mathrm{ce}}^{\prime}$ for $p(r)=2$. Hence for $p(r)=2$, $E_{\mathrm{ce}}^{\prime}$ is the radiation energy of the charge-excess emission $E_{\mathrm{ce}}$. A visualization of $f_{\mathrm{ce}}$ is shown in Fig. 4c.

To determine the optimal parameters of $f_{\mathrm{ce}}$, we follow the same procedure as in the geomagnetic case. For small distances to $X_{\max }$, the signal distribution is maximal at the shower axis which 

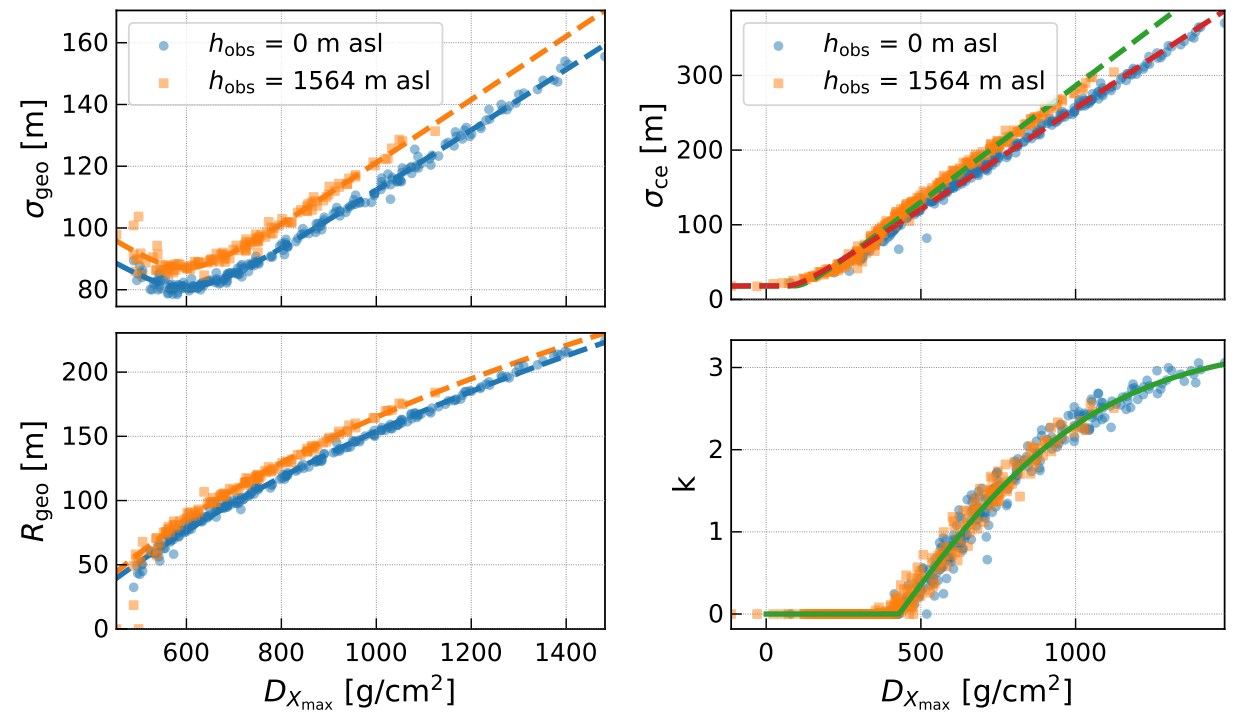

Figure 5: Dependence of fit parameters on the distance to the shower maximum in Eq. (4.1) and (5.1).

is described with $k=0$ (cf. Fig. 2). For larger distances to $X_{\max }, k$ becomes larger than zero to model the observed behavior (cf. Fig. 3). We find that a modification of the exponent $p(r)$ leads to better results for all different distances to $X_{\max }$.

\section{Dependence of fit parameters on the distance to the shower maximum}

The modification parameters $r_{\text {cut }}$ and $b$ of Eq. (4.2) can be parametrized as a function of $D_{\mathrm{X}_{\max }}$ for both the geomagnetic and charge-excess functions. Hence, $f_{\text {geo }}$ and $f_{\text {ce }}$ can be described with three parameters each. We can reduce the number of parameters further by noting that $E_{\mathrm{ce}}$ can be expressed as a function of $E_{\text {geo }}$ using the result of [1]. The remaining parameters depend on the distance to $X_{\max }$. The correlation is presented in Fig. 5. The data set is subdivided into observations at $1564 \mathrm{~m}$ asl and at sea level. For the geomagnetic emission, the parameters $\sigma_{\text {geo }}$ and $R_{\text {geo }}$ (Eq. (4.1)) show a clear correlation with $D_{\mathrm{X}_{\max }}$ if the radio emission of the shower has completed when reaching the observer $\left(D_{\mathrm{X}_{\max }} \approx 450 \mathrm{~g} / \mathrm{cm}^{2}\right)$. The dependence of $\sigma_{\text {geo }}$ can be described with a hyperbola and $R_{\text {geo }}$ with a second order polynomial of the logarithm of $D_{\mathrm{X}_{\max }}$. Both parameters show a slight offset for different observation heights which can be explained by differences in the refractive index.

The parameters $\sigma_{\text {ce }}$ and $k$ of $f_{\text {ce }}$ (Eq. (5.1)) show a correlation to $D_{\mathrm{X}_{\max }}$ over the full $D_{\mathrm{X}_{\max }}$ range. The parameter $\sigma_{\mathrm{ce}}$ is again described with a hyperbola and the parameter $k$ scales with $\sqrt{D_{\mathrm{X}_{\max }}}$.

\section{Combination to 2D function}

Using the relations $f_{\vec{v} \times \vec{B}}(\vec{r})=\left(\sqrt{f_{\text {geo }}(r)}+\cos \phi \sqrt{f_{\text {ce }}(r)}\right)^{2}$ and $f_{\vec{v} \times(\vec{v} \times \vec{B})}(\vec{r})=\sin ^{2} \phi f_{\text {ce }}(r)$ with $\phi=\arctan 2(y, x)$ (cf. Fig. 1), and $f=f_{\vec{v} \times \vec{B}}+f_{\vec{v} \times(\vec{v} \times \vec{B})}$, the geomagnetic and charge-excess energy fluences can be combined to the energy fluence at any position. See [1] for a derivation of 


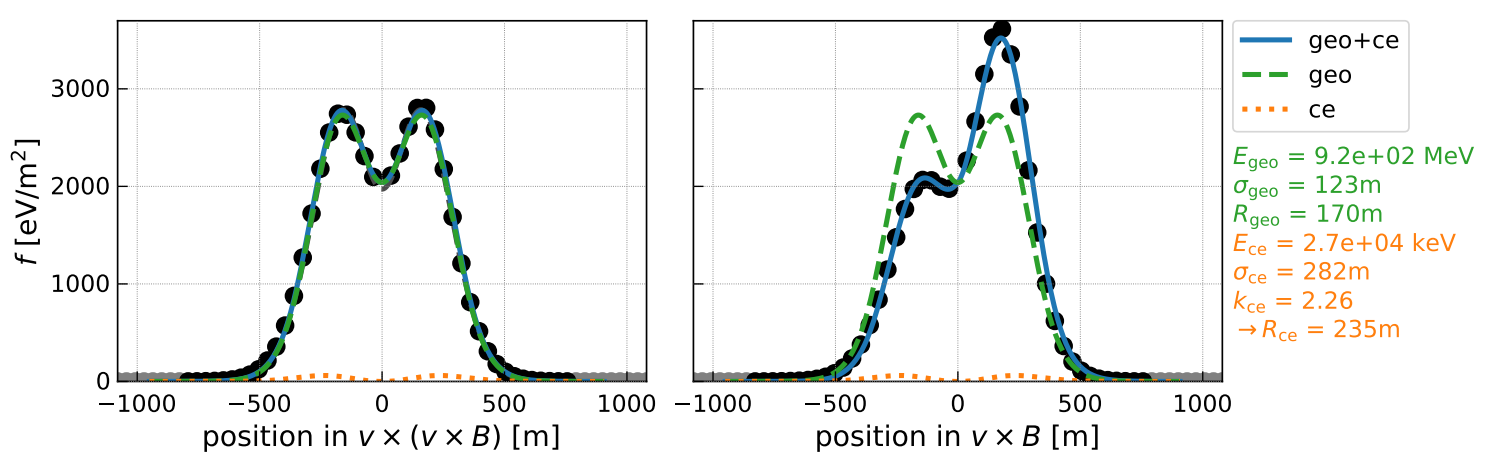

Figure 6: Two-dimensional signal distribution from the combination of the radially symmetric geomagnetic and charge-excess functions. Energy fluence along the $\vec{v} \times(\vec{v} \times \vec{B})$ axis (left) and along the $\vec{v} \times \vec{B}$ axis (right) where the two emission mechanisms interfere constructively (destructively) on the right (left) side of the shower axis.

these relations. In Fig. 6, the energy fluence along the $\vec{v} \times(\vec{v} \times \vec{B})$ and the $\vec{v} \times \vec{B}$ axis is presented. This demonstrates that the observed asymmetry along the $\vec{v} \times \vec{B}$ axis is modeled by the interference between geomagnetic and charge-excess emission.

\section{Conclusion}

We have presented a new analytic model of the radio signal distribution that models the spatial distribution of the energy fluence originating from the two emission mechanism separately. Thereby, we explicitly take into account the polarization of the radio signal by separately describing the $\vec{v} \times \vec{B}$ and $\vec{v} \times(\vec{v} \times \vec{B})$ component of the energy fluence. Hence, the available information at a single antenna station is doubled which allows for a more precise determination of the signal distribution compared to previous models.

One parameter of our model is the radiation energy, which directly relates to the electromagnetic shower energy. The remaining parameters show correlations with the distance to the shower maximum, if the air shower has emitted all its radiation energy before reaching the observer. Hence, we can formulate our model to depend only on the radiation energy, the distance to $X_{\max }$ and the core position which was always at the origin in our simulation study. Thus, our model provides a direct access to the main air-shower parameters energy and $X_{\max }$ and manages to use the smallest possible number of parameters. In the future, it will allow for an improved reconstruction of air showers at radio cosmic-ray observatories.

\section{References}

[1] C. Glaser et al., J. Cosmol. Astropart. Phys. 09(2016)024.

[2] A. Aab et al. (Pierre Auger Collaboration), Phys. Rev. Lett. 116, 241101 (2016).

[3] A. Aab et al. (Pierre Auger Collaboration), Phys. Rev. D 93, 122005 (2016).

[4] A. Nelles et al., Astropart. Phys. 60, 13 - 24 (2015).

[5] T. Huege et al., AIP Conf. Proc. 1535, 128 -132 (2013). 